\title{
Diagnóstico clínico e histopatológico de paratuberculosis bovina en un hato lechero en Colombia
}

\section{Clinical and histopathological diagnosis of bovine paratuberculosis in a dairy herd in Colombia}

\author{
Nicolás Ramírez V, ${ }^{1 *}$ Ph.D(c), Berardo Rodríguez, ${ }^{1}$ Ph.D, Jorge Fernández $\mathrm{S},{ }^{1} \mathrm{Ph} . \mathrm{D}(\mathrm{c})$.
}

\begin{abstract}
${ }^{1}$ Universidad de Antioquia, Facultad de Ciencias Agrarias, Escuela de Medicina Veterinaria, Grupo Centauro, Medellín, Colombia. *Correspondencia: nicoramirez2010@gmail.com.
\end{abstract}

Recibido: Agosto de 2010: Aceptado:Julio de 2011.

\section{RESUMEN}

Objetivo. Analizar retrospectiva y sistemáticamente los hallazgos clínicos e histopatológicos de paratuberculosis bovina Mycobacterium avium subsp. Paratuberculosis (MAP). Los datos fueron obtenidos en diferentes momentos durante un periodo de 8 años (2000-2008) en un hato lechero en Colombia. Materiales y métodos. Se analizó la información documental en 5 casos compatibles con paratuberculisis bovina, así como la información procedente de otros estudios efectuados en el hato sobre la enfermedad realizados paralelamente en el periodo 2000-2008. Resultados. Los 5 animales afectados, presentaron diarrea crónica intermitente, disminución en la producción de leche, enflaquecimiento progresivo, apetito normal, consumo aumentado de agua y constantes fisiológicas normales. A la necropsia se observó engrosamiento de la mucosa intestinal del íleon y de la porción proximal del intestino grueso con múltiples levantamientos y depresiones, que no desaparecían al estirar el tejido. Los vasos sanguíneos mesentéricos se encontraron dilatados y congestivos. Los ganglios linfáticos mesentéricos se encontraron aumentados hasta tres veces, sin clara delimitación de la corteza y de la médula. Las alteraciones histológicas fueron enteritis y linfadenitis granulomatosa. En tres de los animales se evidenciaron abundantes bacilos ácido alcohol resistentes (BAAR) intracelulares en macrófagos, células gigantes y en el intersticio a la coloración de Ziehl-Neelsen. En otros tejidos evaluados no se encontró inflamación de tipo granulomatoso. Conclusiones. Los criterios diagnósticos empleados, así como el análisis de la información diagnóstica generada en otros estudios, permiten confirmar la presencia, circulación y mantenimiento del Mycobacterium avium subsp. paratuberculosis en el hato con un aparente número elevado de animales infectados.

Palabras clave: Enfermedades en bovinos, Mycobacterium avium, paratuberculosis (Fuente: $C A B$ ). 


\section{ABSTRACT}

Objective. To analyze retrospectively and systematicaly the histological and clinical findings of bovine paratuberculosis Mycobacterium avium subsp. Paratuberculosis (MAP). The data were obtained at different times over a period of 8 years (2000-2008) in a dairy herd in Colombia. Materials and methods. Historical data of clinical and histopathological findings, in five cases compatible with bovine paratuberculosis, were studied. Additional information was also analysed from other studies related to the disease in the herd in the same period of time. Results. The infected animals showed intermittent chronic diarrhea, decreased production of milk, progressive weight loss, normal appetite, increased water consumption and normal physiological constants. The necropsy revealed variable thickening of the intestinal mucous membrane in the ileum and proximal portion of the large intestine with multiple uprisings and depressions, which did not disappear in the stretched tissue. Mesenteric vessels were dilated and congestive. Mesenteric lymph nodes were found increased up to three times, without clear delineation of bark and cord. Histological alterations were enteritis and lymphomatoid granulomatosis. Three animals showed abundant acid alcohol resistant Bacilli (AARB) in macrophages, giant cells and in the interstitial tissue by using the Ziehl-Neelsen staining. However, it was not found the granulomatous inflammation in other tissues evaluated. Conclusion. Diagnostic based on clinical, histopathological and necropsy findings as well as on analysis of diagnostic information from other studies, allow us to confirm the presence, circulation and permanence of MAP in the herd with an apparent high number of infected animals.

Key words: Cattle diseases, Mycobacterium avium, paratuberculosis (Source: CAB).

\section{INTRODUCCIÓN}

La paratuberculosis o enfermedad de Johne es causada por el Mycobacterium avium subsp. paratuberculosis (MAP), un patógeno intracelular, ácido-alcohol resistente, facultativo y dependiente de micobactina para su crecimiento in vitro (1). La enfermedad de Johne es una severa gastroenteritis crónica granulomatosa con linfangiectasis y linfangitis asociada, cuya consecuencia final es la aparición de un típico síndrome de malnutrición con pérdida crónica y progresiva de peso y diarrea crónica o intermitente (2-4).

La paratuberculosis causa grandes pérdidas económicas a la industria ganadera. En los hatos de ganado lechero la paratuberculosis ocasiona pérdidas económicas, debidas principalmente a los efectos negativos sobre la producción, la salud y la reproducción; y a causa de los efectos derivados del desmejoramiento de la condición corporal, el descarte prematuro y el costo de los procedimientos diagnósticos y de monitoreo (5). En hatos con una prevalencia relativamente alta de paratuberculosis $(>10 \%)$ se ha reportado una pérdida de hasta $700 \mathrm{~kg}$ de leche por vaca, el descarte de un mayor número de vacas con menores rendimientos por vaca descartada, y una mayor mortalidad en este grupo de edad, en comparación con hatos negativos a la enfermedad (6).

Sin embargo, estudios recientes, que han analizado la relación entre la infección por paratuberculosis y la producción de leche, han demostrado, desde el punto de vista macroeconómico, que una reducción de la enfermedad de Johne en los hatos podría desencadenar un aumento en la producción, y por lo tanto una disminución de su precio, lo que llevaría a una reducción del superávit del productor, pero un aumento del llamado "superávit" del consumidor, quien podría acceder a un producto más económico $(7,8)$.

Cada animal con enfermedad de Johne entra en una de cuatro categorías o estados de la enfermedad. Estos se establecen de acuerdo con la severidad de 
los signos clínicos, la eliminación potencial de organismos en el ambiente y la probabilidad de detección de la enfermedad usando métodos corrientes de laboratorio (9). Estado I o infección "silenciosa", en este estado se encuentran animales hasta 2 años de edad que no presentan ningún signo clínico y aparentemente son iguales al resto de los animales, la infección sólo es detectable en sus tejidos por cultivo o examen histopatológico y aunque eliminan el agente por vía fecal, las cantidades de microorganismos están por debajo del umbral de detección por las pruebas de rutina (9).

Estado II o enfermedad subclínica, los animales con infección subclínica por MAP no presentan signos de enfermedad de Jhone, pero pueden tener niveles detectables de anticuerpos contra MAP, eliminan el agente por heces y pueden ser detectados por lo medios de cultivo. La mayoría de ellos no son detectados por las técnicas usadas corrientemente y algunos avanzan al estado III de la afección (9).

Estado III o enfermedad clínica, se presenta después de un periodo de incubación que dura de 2 a 10 años. Incluye la pérdida gradual de peso a pesar de un apetito normal $o$ en ocasiones aumentado. Durante un periodo de pocas semanas la consistencia de la materia fecal es más fluida y esto concuerda con la pérdida de peso, la diarrea puede ser intermitente al comienzo con periodos de consistencia normal de la materia fecal. Usualmente el animal incrementa el consumo de líquido. Los signos vitales como frecuencia cardiaca, respiratoria y temperatura son normales (9).

Estado IV o enfermedad clínica avanzada, a medida que la enfermedad progresa el animal incrementan la letargia, debilidad y emaciación. El edema intermandibular debido a la hipoproteinemia es típico en enfermos avanzados. La caquexia y la diarrea caracterizan los estados terminales de la enfermedad. Muchos animales son eliminados del hato antes de este estado debido a la disminución en la producción de leche, pérdida severa de peso o ambos.
Una vez la diarrea se hace profusa y la hipoproteinemia severa con edema submandibular, la condición del animal se deteriora rápidamente, a veces en cuestión de días. Otras veces la muerte ocurre como resultado de la deshidratación y la caquexia (9).

La enfermedad de Johne en los bovinos se caracteriza por una transmisión temprana vía oro-fecal por ingestión del microorganismo en la leche, alimentos o superficies contaminados (2). La contaminación del pasto, los alimentos y los utensilios se ha descrito como otros factores de vehiculización y contagio de la enfermedad. La enfermedad clínica se da sobre todo entre los 3 y los 5 años de edad y se considera que la etapa clínica puede durar de 3 a 6 meses (10). MAP se ha asociado con la presentación de la enfermedad de Crohn en humanos; sin embargo, no se ha podido demostrar su participación como agente etiológico, y se ha sostenido una controversia científica sobre su relación con esta enfermedad (11).

El diagnóstico temprano de paratuberculosis es difícil debido a su largo periodo de incubación y a su lenta progresión. Para el diagnóstico de MAP en bovinos se utilizan diferentes pruebas tales como la detección de anticuerpos contra MAP, la detección de genes de MAP, el cultivo bacteriano de materia fecal y el examen para detección de MAP en muestras de tejido $(9,12)$, asi como prueba de diagnóstico basada en hipersensibilidad cutánea retardada, denominada también prueba de Johnina intradérmica (13). Dentro de los test para la detección de anticuerpos contra MAP, la detección por medio de ELISA (del ingés enzyme-linked immunosorbent assay) es el de mayor disponibilidad y el más ampliamente utilizado para establecer la situación de la paratuberculosis en los hatos (9). Sin embargo, esta prueba presenta limitaciones relacionadas con la baja sensibilidad, debido principalmente a su lenta progresión, lo que no garantiza una adecuada capacidad de detección en animales en una etapa temprana (2). La detección de genes de MAP por medio de la técnica de PCR (del inglés Polymerase 
Chain Reaction) presenta ventajas (rapidez, identificación del agente) y desventajas (sensibilidad y especificidad moderada) $(2,9)$.

El aislamiento de MAP por cultivo de materia fecal se considera la prueba diagnóstica de oro de la paratuberculosis (1). La prueba basada en hipersensibilidad cutánea retardada ha sido utilizada con éxito para el diagnóstico de paratuberculosis, y tiene la ventaja de ser una prueba fácil de aplicar y de leer en el hato $(13,14)$. Sin embargo, la prueba se considera de baja especificidad (15). El diagnóstico histopatológico de paratuberculosis es un método ampliamente utilizado y sugerido para la identificación de animales infectados y afectados por la enfermedad, y para evaluar la persistencia de MAP y la severidad de la respuesta inmune mediada por células en el hospedero (3).

Aunque la enfermedad de Johne se ha investigado en Colombia desde hace más de 60 años, se considera que el comportamiento epidemiológico de la enfermedad en el país es desconocido. En el 2000 y durante los años subsiguientes varios bovinos adultos de la raza Holstein pertenecientes a un hato lechero en el departamento de Antioquia (Colombia), presentaron diarrea crónica, disminución en la producción de leche y enflaquecimiento progresivo compatible con paratuberculosis bovina. Los casos compatibles con paratuberculosis se presentaron siempre de manera individual y esporádica a lo largo del tiempo. Varios estudios diagnósticos realizados a diferentes animales en ese hato entre 2000 y 2010, han proporcionado resultados que indican la presencia de MAP en el rebaño (16-18). A pesar del seguimiento de los casos compatibles y los exámenes realizados a los animales del hato, los resultados de diagnóstico clínico e histopatológico obtenidos durante el periodo de tiempo 2000-2008 no se habían analizado sistemáticamente hasta la fecha. El objetivo del presente trabajo fue el análisis retrospectivo y sistemático de los resultados de diagnóstico clínico e histopatológico de paratuberculosis bovina, obtenidos en diferentes momentos durante un período de 8 años en un hato lechero en Colombia.

\section{MATERIALES Y MÉTODOS}

Aval comité de Ética. Esta investigación consistió en un estudio retrospectivo que se originódelseguimiento clínicoyepidemiológico de bovinos naturalmente enfermos durante 8 años en una granja perteneciente a la Universidad de Antioquia. Durante su ejecución no se realizaron procedimientos experimentales, por esta razón no se solicitó aval de un comité de ética. Las eutanasias fueron realizadas por motivos humanitarios para evitar el sufrimiento de los animales; sin embargo, se respetaron los principios bioéticos establecidos internacionalmente (19). Las necropsias se efectuaron como una medida de bioseguridad para realizar un diagnóstico que permitiera identificar los posibles riesgos para la salud del hato y para la salud pública.

\section{Animales, datos generales y lugar} de estudio. Los datos analizados retrospectivamente en este estudio fueron recopilados de la información documental generada durante el seguimiento y diagnóstico clínico, el diagnóstico histopatológico, así como los resultados de otros estudios diagnósticos realizados paralelamente en el periodo 2000-2008 en un hato lechero ubicado en el Municipio de San Pedro de los Milagros, departamento de Antioquia, Colombia. El hato está ubicado en una zona de vida de Bosque Húmedo Montano Bajo (bh-MB), con una temperatura promedio de $15^{\circ} \mathrm{C}$, máxima de $22^{\circ} \mathrm{C}$ y mínima de $7^{\circ} \mathrm{C}$. Altura sobre el nivel del mar entre 2350 a 2500 metros, precipitación anual de 1575 milímetros y humedad relativa del $72 \%$ (20). En la hacienda donde está ubicado el hato, existen además una unidad de producción avícola y una unidad de produccion porcina. La población bovina estaba conformada por 105 bovinos hembra de la raza Holstein y BlancoOrejinegro (BON) x Holstein alimentados con pasto Kikuyo (Pennisetum clandestinum), concentrado comercial, sal a voluntad y agua. Los animales se vacunan contra fiebre aftosa, brucelosis, carbón sintomático, edema maligno y septicemia hemorrágica. El hato 
es libre de tuberculosis bovina, de acuerdo con el seguimiento sanitario efectuado por el Instituto Colombiano Agropecuario, entidad nacional competente para la certificación en sanidad animal. La desparasitación se efectúa periódicamente de acuerdo con los resultados del exámen coprológico.

Evaluación clínica. Durante el periodo de estudio cinco animales con síntomas compatibles con paratuberculosis se evaluaron clínicamente, en ellos se registró la temperatura, la frecuencia cardíaca y la frecuencia respiratoria. Además, se determinó la condición corporal (21), el estado reproductivo y la presencia de diarrea crónica.

\section{Evaluación de algunos parámetros hemáticos, de química sanguínea y de resultados de la prueba de hipersensibilidad cutánea retardada.} Se analizaron los únicos datos disponibles de algunas pruebas como el hemograma completo, nitrógeno uréico en sangre (BUN), aspartato amino transferasa (AST), creatinina y glucosa. También, se analizaron los resultados que se tenían de la prueba de hipersensibilidad cutánea retardada.

Pruebas serológicas y parasitológicas. Adicionalmente, en el período de estudio y previamente a la presentación de los síntomas compatibles con paratuberculosis, se efectuaron pruebas de ELISA en suero sanguíneo a cuatro de los bovinos del estudio con el fin de detectar anticuerpos contra rinotraqueítis infecciosa bovina (IBR) (Chekit Trachitest; Bommli, Liebefeld-Bern, Switzerland), diarrea viral bovina (DVB) (Herd - Chek, PPRS 2XR; IDEXX Laboratories, Bancelona, Spain) y leucosis viral bovina enzootica (LVBE) (Chekit Leucotest, Bommeli, AG, Bern Switzerland).

Biopsia. A un animal al momento de la aparición de los síntomas compatibles, al bovino 3, (Tabla 1), se le tomó biopsia de intestino delgado con abordaje quirúrgico por el flanco derecho, con el fin de realizar diagnóstico histopatológico antemortem de paratuberculosis, posteriormente la condición del animal empeoró y se le practicó necropsia.

Tabla 1. Parámetros clínicos y de laboratorio de 5 bovinos con síntomas compatibles con paratuberculosis en un hato lechero de Colombia.

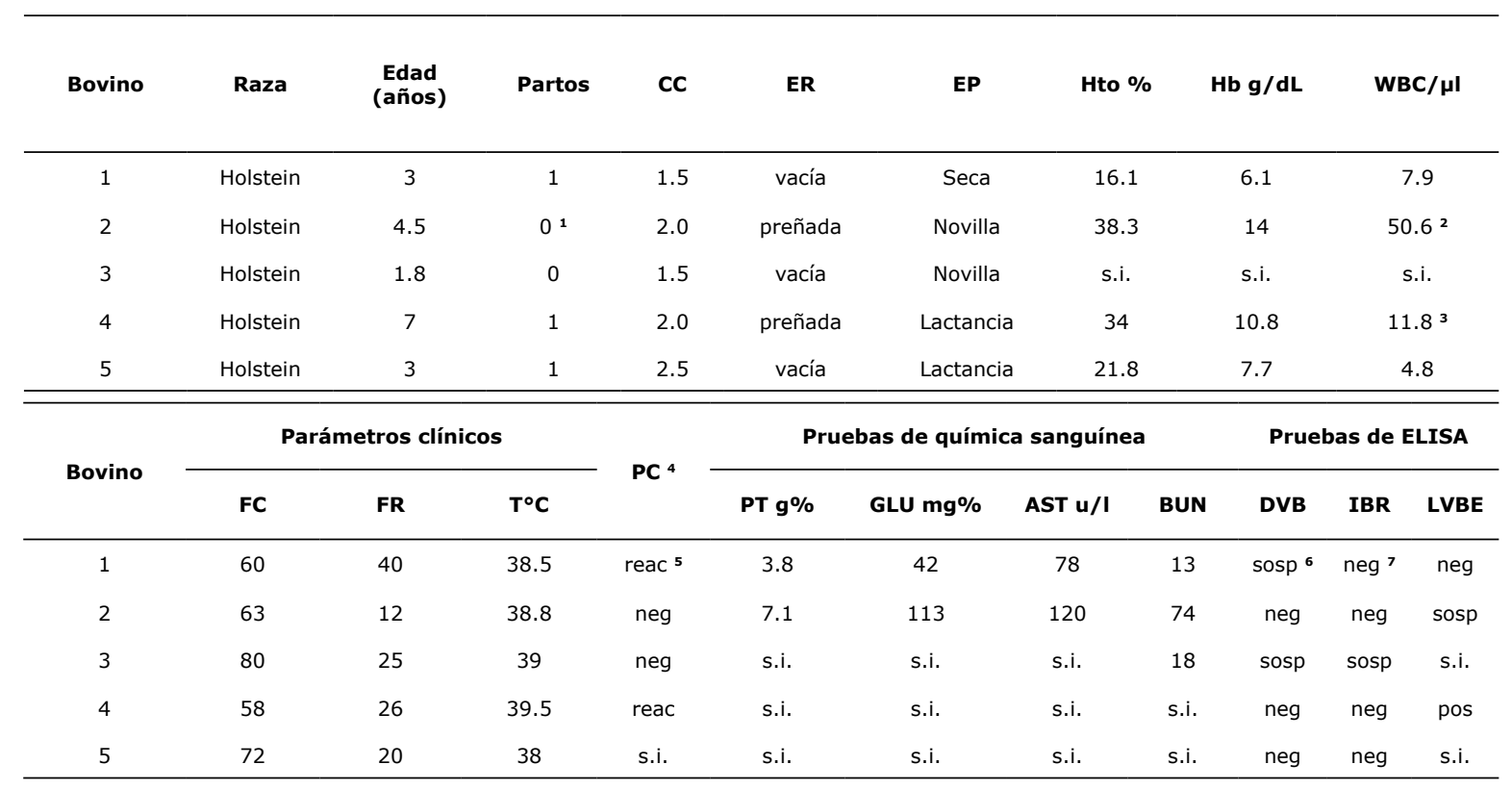

$\mathrm{CC}=$ Condición corporal; $\mathrm{ER}=$ Estado reproductivo; $\mathrm{EP}=$ Estado productivo; Hto=Hematocrito; Hb=Hemoglobina; WBC=Conteo leucocitario blancas; $\mathrm{FC}=$ Frecuencia cardíaca; $\mathrm{FR}=$ Frecuencia respiratoria; $\mathrm{T}=$ Temperatura; $\mathrm{PC}=\mathrm{Prueb}$ a cutánea; $\mathrm{PT}=\mathrm{Proteínas} \mathrm{totales;} \mathrm{GLU}=\mathrm{Glucosa}$; $\mathrm{AST}=$ Aspartato amino transferasa; $\mathrm{BUN}=$ Nitrógeno ureico en sangre; DVB=Diarrea viral bovina; IBR=Rinotraqueitis infecciosa bovina; LVBE=Leucosis viral bovina enzootica; ${ }^{1}$ Presentó dos abortos; ${ }^{2}$ Presentó leucocitosis con linfocitosis; ${ }^{3}$ Presentó linfocitos atípicos; ${ }^{4}$ Prueba comparativa, según Ramírez et al (16); ${ }^{5}$ Reactor; ${ }^{6}$ Sospechoso; 7 Negativo. 
Necropsia. A los cinco animales con síntomas compatibles con paratuberculosis, que en su momento presentaron una precaria condición general, sin respuesta a ningún tratamiento o paliativo, se les efectuó eutanasia en condiciones humanitarias con sulfato de magnesio, previa sedación profunda con xilacina y acepromacina, siguiendo los principios propuestos por "The International Guiding Principles for Biomedical Research Involving Animals" (19) y se les practicó la necropsia de acuerdo con protocolos previamente descritos (22). Durante las necropsias se tomaron fragmentos de intestino delgado y grueso, ganglios linfáticos mesentéricos, pulmón, hígado y riñón, los cuales fueron fijados en formalina tamponada al $10 \%$.

Histopatología. Los tejidos recolectados en la necropsia de cuatro animales y la biopsia de intestino del bovino 3, fueron procesados en el Laboratorio de Patología Animal de la Universidad de Antioquia en Medellín (Colombia), por medio técnicas histológicas convencionales. Los tejidos fueron deshidratados en concentraciones crecientes de alcohol, aclarados con xilol, incluidos en parafina, cortados a $4 \mu \mathrm{m}$ de espesor y se realizó tinción con Hematoxilina-eosina y Ziehl-Neelsen para caracterizar las lesiones microscópicas y para detectar bacterias ácido-alcohol resistentes, respectivamente. El análisis histopatológico de las placas se efectuó utilizando microscopía de luz. La detección de los microorganismos ácidoalcohol resistentes se realizó mediante el examen con objetivo de inmersión bajo un aumento de 1000X.

\section{RESULTADOS}

Hallazgos generales. Los cinco animales afectados tenían edades que oscilaban entre 1.8 y 7 años y presentaban diferentes estados reproductivos y condiciones corporales al momento de presentar las manifestaciones clínicas compatibles con paratuberculosis. En la tabla 1 se presentan las características generales de los animales estudiados, los parámetros clínicos (frecuencia cardíaca, frecuencia respiratoria y temperatura) así como los resultados del hemograma, química sanguínea, la prueba comparativa (PC) y de
ELISA para IBR, DVB y LVBE. Los resultados de necropsia y examen histopatológico se presentan en la tabla 2.

Tabla 2. Hallazgos histopatológicos macro y micro de 5 bovinos con síntomas compatibles con paratuberculosis en un hato lechero de Colombia.

\begin{tabular}{|c|c|c|c|c|}
\hline \multirow[b]{2}{*}{ Bovino } & \multirow{2}{*}{$\begin{array}{c}\text { Mucosa } \\
\text { intestinal } \\
\text { engrosada } \\
\text { y plegada }\end{array}$} & \multicolumn{3}{|c|}{ Examen histopatológico } \\
\hline & & Intestino & $\begin{array}{c}\text { Ganglio } \\
\text { linfático } \\
\text { mesentérico }\end{array}$ & $\begin{array}{c}\text { Ziehl-Neelsen } \\
\text { BAAR }\end{array}$ \\
\hline 1 & presente & IG & IG & Positivo GL, I \\
\hline 2 & presente & IG & s.i. ${ }^{1}$ & s.i. \\
\hline 3 & $\begin{array}{c}\text { presente } \\
\text { (necropsia) }\end{array}$ & $\begin{array}{c}\text { IG } \\
\text { (biopsia) }\end{array}$ & $\begin{array}{l}\text { Positivo I } \\
\text { (biopsia) }\end{array}$ & $\begin{array}{c}\text { s.i. } \\
\text { (necropsia) }\end{array}$ \\
\hline 4 & presente & IG & IG & negativo \\
\hline 5 & presente & IG & IG & Positivo I y $\mathrm{H}$ \\
\hline
\end{tabular}

Hallazgos clínicos. Los cinco bovinos afectados en el periodo de análisis presentaron algunos aspectos clínicos en común como diarrea crónica y enflaquecimiento, el bovino 1 presentó diarrea con sangre, ver tabla $1 \mathrm{y}$ figura 1. Asimismo los animales presentaron apetito normal y constantes fisiológicas (temperatura, frecuencia cardíaca y respiratoria) dentro de los rangos normales (23). En el examen coprológico de los cinco animales se determinó que presentaban una baja carga parasitaria gastrointestinal. Los exámenes de BUN, AST, glucosa, y hemograma completo, arrojaron resultados así: El caso 1 presentó hipoproteinemia (3.8 $\mathrm{g} / \mathrm{dL})$ y anemia, mientras que el caso 2 presentó marcada leucocitosis con linfocitosis y aumentó en los niveles de glucosa, AST y BUN según Gasque, 2008 (23), el caso 4 presentó linfocitos atípicos. Los demás resultados estaban dentro de los rangos normales.

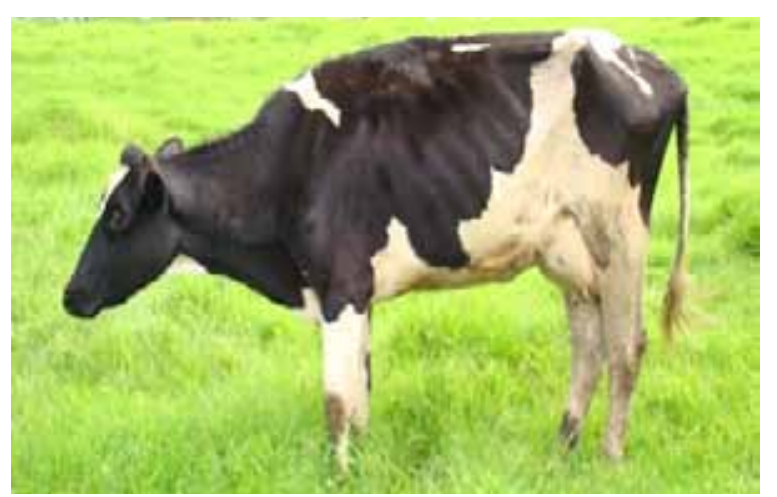

Figura 1. Animal con diarrea crónica y enflaquecimiento progresivo (bovino 5). 
Hallazgos de necropsia. En todos los casos evaluados se observó severa emaciación de los animales, engrosamiento variable de la mucosa intestinal del íleon y de la porción proximal del intestino grueso, especialmente ciego y parte anterior del colon (Tabla 2). El aspecto de la mucosa era brillante, tumefacto y congestiva, con múltiples levantamientos y depresiones semejantes a las circunvoluciones del cerebro, que no desaparecían al estirar el tejido (Figura 2).

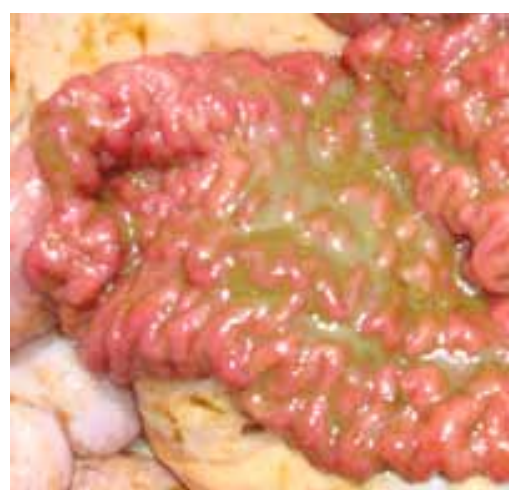

Figura 2. Mucosa del ileón (bovino 5).

Los vasos sanguíneos mesentéricos se encontraban dilatados y congestivos. Los ganglios linfáticos mesentéricos se encontraron aumentados hasta tres veces el tamaño normal, en su superficie de corte no se evidenció una clara delimitación de la corteza ni de la médula. La corteza presentó coloración amarillenta brillante que se hacía parda hacia la médula y en el tejido subcapsular.

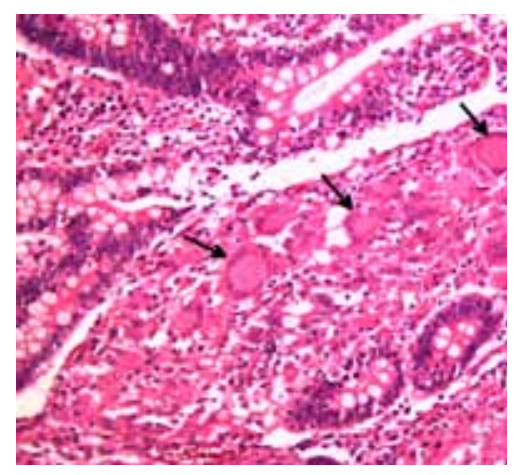

Figura 3. Íleon. Inflamación granulomatosa, células epitelioides y células gigantes multinucleadas (flechas). H-E.
Hallazgos histopatológicos. Las alteraciones histológicas comunes a todos los animales evaluados fueron enteritis y linfadenitis granulomatosa. Estas lesiones se caracterizaron por la erosión epitelial en la mucosa de los intestinos, atrofia y fusión de las vellosidades, y la presencia de un infiltrado difuso o multifocal en la lámina propia y en la submucosa, constituido principalmente por linfocitos, plasmocitos, macrófagos, células epitelioides y células gigantes multinucleadas tipo Langhans. En tres de los animales se evidenciaron con la coloración de Ziehl-Neelsen, abundantes bacilos ácido alcohol resistentes (BAAR) intracelulares en macrófagos, en células gigantes y en el intersticio principalmente en la lámina propia.

En los ganglios mesentéricos se observó inflamación granulomatosa con distribución variable, ocasionalmente difusa 0 multifocal involucrando los senos subcapsulares, peritrabeculares y medulares. Esta inflamación, al igual que en el intestino, se caracterizó por la presencia de linfocitos, plasmocitos, macrófagos, células epitelioides, células gigantes multinucleadas tipo Langhans (Figura 3) y en algunos de los casos presencia de BAAR (Figura 4). En otros tejidos evaluados como hígado, pulmón, bazo, estomago, riñón, vejiga, arteria, retículo y omaso, no se encontró inflamación de tipo granulomatoso.

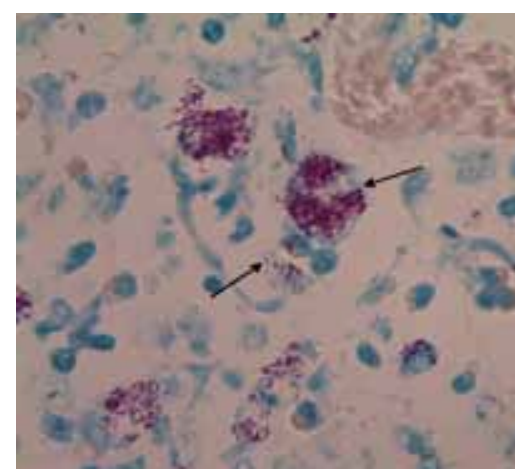

Figura 4. Íleon. Bacilos ácido alcohol resistentes en la mucosa (flechas). Coloración de Ziehl Neelsen. 


\section{DISCUSIÓN}

En este artículo se presenta el reporte diagnóstico de paratuberculosis bovina basado en los resultados del examen clínico, necropsia e histopatología de 5 animales pertenecientes a un hato lechero colombiano. La mayoría de los signos clínicos observados, así como los hallazgos de necropsia e histopatologicos, coinciden con los descritos para la infección por MAP en el ganado vacuno, y se consideran característicos de un estado de enfermedad clínica (estado III), o en algunos casos de enfermedad clínica avanzada (estado IV) (24).

Cuatro de los cinco animales afectados, se encontraban en el rango de edad compatible con la presentación de la enfermedad de Johne en bovinos (24). El hallazgo del bovino 3 (1.8 años de edad), contrasta con el conocimiento generalmente aceptado de que en bovinos no se observan signos clínicos antes de dos años de edad (25). A pesar de esto, y aunque la evidencia acerca de la mayor suceptibilidad a MAP se concentra en los primeros meses de edad del ternero, no puede descartarse que algunos animales, especialmente en hatos con una alta carga de MAP en el ambiente y con deficientes prácticas de manejo, puedan infectarse y desarrollar la enfermedad clínica con una edad inferior a 2 años (26). La biopsia de ganglio linfático ileal para examen histopatológico se considera de gran utilidad por la detección precoz que provee, y por ser sumamente específica y sensible (3), a pesar de la desventaja que implica una intervención quirúrgica. En este sentido la biopsia de intestino delgado efectuada al bovino 3 , se convirtió en una técnica diagnóstica útil para comprobar el padecimiento de la enfermedad en este caso, que por su edad generaba dudas en su diagnóstico.

La relativa baja condición corporal de los animales estudiados $(1.5$ - 2.5) se asocia normalmente con la diarrea crónica intermitente, típica de la etapa clínica avanzada de la enfermedad en la que se encontraban al momento de la eutanasia (24), aunque existen reportes de animales con relativa buena condición corporal $(\geq 2.75)$, que han resultado positivos a MAP en el cultivo de ganglio e intestino (27). Tales hallazgos sugieren la independencia entre la infección por MAP y el detrimento de la condición corporal en fases anteriores a la fase clínica avanzada, pero no corresponden a los efectos negativos sobre la condición corporal en animales clínicamente enfermos como en los de este estudio, los cuales presentaron inclusive apetito normal.

Debido al reducido número de casos analizados y a la falta de información acerca de la relación entre paratuberculosis y gestación, es difícil emitir conclusiones acerca del efecto del estado reproductivo (vacía/preñada) de los animales estudiados sobre la presentación de la enfermedad. En todo caso existen evidencias de que el periparto es un proceso dinámico para la inmunidad de la vaca, que potencialmente altera la habilidad del animal para impedir la infeccion por MAP (28), lo que podría explicar por qué la mayoría de las vacas afectadas de este estudio tenían al menos un parto y/o se encontraban en gestación al momento de la necropsia. En este estudio llama la atención que la gestación no provocó la disminución de la diarrea, como ha sido planteado en la literatura (26).

Todos los animales estudiados tenían ninguno o máximo un parto al momento de la presentación de los síntomas y en la mayoría de los casos, excepto el caso 3, se trataba de animales adultos (Tabla 1). Teóricamente animales sin partos, de un solo parto o de un bajo número de partos tienen menor riesgo de resultar positivos en algunas pruebas diagnósticas (e.g. ELISA) (29), lo que puede reforzar la interpretación de que se trata de un hato altamente contaminado con MAP, que propicia la presentación constante de casos clínicos.

A pesar de que todos los animales presentaron signos clínicos y lesiones postmmortem compatibles con paratuberculosis, sólo dos animales reportados en el presente estudio (bovino 1 y 4), habían resultado positivos a la tuberculina y a la PC en un estudio preliminar (16), lo que sugiere que existe un bajo nivel de asociación entre lesiones de paratuberculosis y positividad a la prueba de tuberculina, que concuerda con lo comunicado por otros autores (30). 
En un estudio reciente se confirmó que el bovino 5 efectivamente se encontraba infectado con MAP, según los resultados de la prueba de PCR de ADN obtenido de cultivo bacteriológico de materia fecal y/o de medio líquido Middlebrook 7H9, inoculado con materia fecal del mismo animal (18). En el análisis de los exámenes diagnósticos realizados a los 5 animales afectados por paratuberculosis, se determinó que dos de ellos (bovinos 1 y 3 ) resultaron sospechosos en ELISA para DVB. Asimismo, se sabe por medio de estudios previos, que el hato es seropositivo para DVB (16) y para MAP (17), lo que es consistente con el conocimiento de que la seropositividad a DVB constituye un factor de riesgo de seropositivdad para MAP (31). De la misma manera, la coinfección con el virus de la LVBE (al igual que el virus de la DVB) se han mencionado como factores causantes de estrés e inmunosupresión (31), lo que pudo favorecer la circulación, transmisión y perpetuación del patógeno en el hato y por consiguiente la presentación esporádica, pero continua de casos clínicos a lo largo del tiempo.

La hipoproteinemia $(3.8 \mathrm{~g} / \mathrm{dl})$ en el bovino 1 , es un hallazgo comúnmente reportado en los casos clínicos de paratuberculosis, y se encuentra asociado a la pérdida permanente de proteínas a causa de la diarrea crónica (4). La anemia marcada reportada igualmente en el bovino 1 , que no es un hallazgo común en la literatura de la enfermedad, se presume relacionada con la diarrea con sangre que presentó la vaca. Este síntoma pudo presentarse por coinfección con agentes parasitarios a pesar del bajo nivel de parasitismo reportado en el control coprológico en los animales estudiados. Asimismo, no se descarta que se haya podido presentar por la coinfección con enterobacterias, asociada a la baja de la inmunidad del animal. El bovino 2 presentó leucocitosis marcada con linfocitosis absoluta, lo cual puede estar relacionado con la posible positividad que tenía al virus de la leucosis enzootica bovina, LVBE, adicionalmente este animal presentó aumento en los niveles de glucosa, aspartato amino transferasa, AST y nitrógeno ureico en sangre, BUN, lo que pone de manifiesto las alteraciones que presentaba el animal en varios órganos como riñones e hígado que concuerdan con los hallazgos a la necropsia de glomerulonefritis crónica y de áreas focales de degeneración turbia hepática. El bovino 4 presentó linfocitos atípicos, muy posiblemente asociado a la seropositividad que presentó el animal a infección por el virus de la LVBE, ver tabla 1.

La severa emaciación de los animales, así como el tipo de lesiones macroscópicas encontradas en los cinco animales a la necropsia y su ubicación, coinciden con las lesiones primarias de paratuberculosis descritas en la literatura (26). Asimismo todos los animales presentaron diarrea posiblemente asociada a esas lesiones intestinales, lo que concuerda con lo reportado por Balseiro et al (30). En otros órganos no se encontraban lesiones evidentes en la inspección macroscópica. Es de resaltar que el bovino 3 de 1.8 años de edad presentó lesiones avanzadas a nivel de intestino típicas de la enfermedad, lo cual de acuerdo a (26) es característico de animales de edades comprendidas, entre 3 y 5 años principalmente.

Las lesiones encontradas a nivel de la mucosa y submucosa del íleon consistieron en una enteritis granulomatosa caracterizada por la presencia de infiltrado inflamatorio constituido por cantidad variable de linfocitos, células plasmáticas, macrófagos, células epitelióides, células gigantes tipo Langhans y presencia de BAAR evidentes con la tinción de Zeehl Neelsen a nivel de intestino y de ganglio (Tabla 2). Estas lesiones son características en el bovino de la paratuberculosis lo cual está en concordancia con lo reportado $(3 ; 30)$. La mayoría de los animales presentó lesiones histopatológicas del tipo difuso intermedio que suele estar asociada con cambios microscópicos y macroscópicos característicos de vacas con síntomas severos de paratuberculosis (3).

El bovino 5 presentó inflamación granulomatosa severa involucrando la lámina propia y la submucosa; causando distorsión y erosión de las vellosidades con presencia abundante de BAAR en el tejido y en las heces, esto sugiere que la severidad de la lesión está relacionada con una infección avanzada caracterizada por eliminación de MAP en heces y presencia de signos clínicos 
como lo ha comunicado Dennis et al (32). El bovino 4, presentó enteritis granulomatosa leve y fue negativo para la coloración de BAAR, lo que sugiere que en lesiones leves la cantidad de microorganismos puede ser menor o presentar una distribución desigual en el tejido, por lo que su detección puede requerir un número elevado de preparaciones histológicas para aumentar la sensibilidad de la técnica.

En conclusión, los criterios diagnósticos empleados basados en exámenes clínico e histopatológicos asi como en hallazgos de necropsia, permiten confirmar la presencia, circulación y mantenimiento del MAP en el hato.

Esta situación demanda controles inmediatos para minimizar en el menor tiempo posible el impacto sanitario y económico de la enfermedad en el ganado bovino del hato, y el potencial efecto nocivo en la salud pública a causa del número de personas que diariamente entran en contacto con estos animales y a la venta y consumo de la leche producida.
La biopsia efectuada a nivel de intestino delgado, puede ser una alternativa a considerar en aquellos casos en los cuales se requiera de la confirmación de la enfermedad en casos individuales.

Debido a que el hato se encuentra ubicado en el altiplano norte, la principal región lechera del departamento de Antioquia, es importante realizar estudios epidemiológicos que permitan caracterizar la situación de esta enfermedad en esta zona, como una primera medida para establecer su verdadero impacto sanitario, económico y social.

\section{Agradecimientos}

Al Departamento de Formación Académica de Haciendas de la Facultad de Ciencias Agrarias de la Universidad de Antioquia, por permitir el desarrollo del estudio en las instalaciones de la granja, y al laboratorio de Patología Animal de la Escuela de Medicina Veterinaria de la Universidad de Antioquia por el análisis de la muestras.

\section{REFERENCIAS}

1. Chacon O, Bermudez LE, Barletta RG. Johne's disease, inflammatory bowel disease, and Mycobacterium paratuberculosis. Annu Rev Microbiol 2004; 58:329-363.

2. Harris NB, Barletta RG. Mycobacterium avium subsp. paratuberculosis in Veterinary Medicine. Clin Microbiol Rev 2001; 14(3):489-512.

3. Gonzalez J, Geijo MV, Garcia-Pariente C, Verna A, Corpa JM, Reyes LE, et al. Histopathological classification of lesions associated with natural paratuberculosis infection in cattle. J Comp Pathol 2005; 133(2-3):184-196.
4. Brady C, O'Grady D, O'Meara F, Egan J, Bassett $\mathrm{H}$. Relationships between clinical signs, pathological changes and tissue distribution of Mycobacterium avium subspecies paratuberculosis in 21 cows from herds affected by Johne's disease. Vet Rec 2008; 162(5):147-152.

5. Hutchinson LJ. Economic impact of paratuberculosis. Vet Clin North Am Food Anim Pract 1996; 12(2):373-381.

6. Ott $S L$, Wells SJ, Wagner BA. Herd-level economic losses associated with Johne's disease on US dairy operations. Prev Vet Med 1999; 40(3-4):179-192. 
7. Losinger WC. Welfare effects of reduced milk production associated with Johne's disease on Johne's-positive versus Johne's-negative dairy operations. J Dairy Res 2006; 73(3):378-384.

8. Losinger WC. Economic impact of reduced milk production associated with Johne's disease on dairy operations in the USA. J Dairy Res 2005; 72(4):425432.

9. Tiwari A, Vanleeuwen JA, McKenna SL, Keefe GP, Barkema HW. Johne's disease in Canada Part I: clinical symptoms, pathophysiology, diagnosis, and prevalence in dairy herds. Can Vet J 2006; 47(9):874-882.

10. Bülte $M$, Schönenbrücher $H$, Abdulmawjood A. From farm to fork--Mycobacterium avium ssp. paratuberculosis (MAP) as zoonotic agent?. Berl Munch Tierarztl Wochenschr 2005; 118(9-10):377-385.

11. Feller M, Huwiler K, Stephan R, Altpeter E, Shang A, Furrer H et al. Mycobacterium avium subspecies paratuberculosis and Crohn's disease: a systematic review and meta-analysis. Lancet Infect Dis 2007; 7(9):607-613.

12. Collins MT, Gardner IA, Garry FB, Roussel AJ, Wells SJ. Consensus recommendations on diagnostic testing for the detection of paratuberculosis in cattle in the United States. J Am Vet Med Assoc 2006; 229(12):1912-1919.

13. Kalis $\mathrm{CH}$, Collins MT, Hesselink JW, Barkema HW. Specificity of two tests for the early diagnosis of bovine paratuberculosis based on cell-mediated immunity: the Johnin skin test and the gamma interferon assay. Vet Microbiol 2003; 97(1-2):73-86.
14. Klawonn $\mathrm{W}$, Cussler $\mathrm{K}$, Dräger KG, Gyra $H$, Köhler $H$, Zimmer $K$, et al. The importance of allergic skin test with Johnin, antibody ELISA, cultural fecal test as well as vaccination for the sanitation of three chronically paratuberculosis-infected dairy herds in Rhineland-Palatinate. Dtsch Tierarztl Wochenschr 2002; 109(12):510-516.

15. Olsen I, Sigurgardottir G, Djonne B. Paratuberculosis with special reference to cattle. A review. Vet Q 2002; 24(1):12-28.

16. Ramírez-Vasquez NF, Gaviria-Botero G, Restrepo-Betancur LF, Gomez-N C. Diagnóstico epidemiológico referente a varias patologías de bovinos en tres haciendas de la Universidad de Antioquia. 2002. [accesado agosto de 2010]. URL disponible en: http://agronica.udea. edu.co/talleres/Medicina/Prof\%20 Nicolas\%20\%20Ram\%C3\%ADrez/ Articulo Proyecto diagnóstico.doc.

17. Fernández-Silva $\mathrm{JA}$, Abdulmawjood $A$, Akineden Ö, Bülte $M$. Serological and molecular detection of Mycobacterium avium subsp. paratuberculosis in cattle of dairy herds in Colombia. Trop Anim Health Prod 2011; 43(8):1501-1507.

18. Zapata R. MM, Arroyave O., Ramirez R., Piedrahita C., Rodas G. JD, Maldonado E. JG. Identification of Mycobacterium avium subspecies paratuberculosis by PCR techniques and establishment of control programs for bovine paratuberculosis in dairy herds. Rev Colomb Cienc Pecu 2010; 23:17-27.

19. Council for International Organizations of Medical Sciences (CIOMS). International Guiding Principles for Biomedical Research Involving Animals, 1985. Geneva: CIOMS; 2011. 
20. Municipio de San Pedro de los Milgros. Sitio Oficial de San Pedro de los Milagros en Antioquia, Colombia. http:// sanpedrodelosmilagros-antioquia. gov.co . 2011. 4-12-2011. [en línea] 2011. [Consultado noviembre de 2011]. URL disponible en: http:// sanpedrodelosmilagros-antioquia.gov. co/index.shtml.

21. Edmonson AJ, Lean IJ, Weaver LD, Farver T, Webster G. A Body Condition Scoring Chart for Holstein Dairy Cows. J Dairy Sci 1989; 72:68-78.

22. Aluja AS, Casas FC. Técnicas de necropsia en animales domésticos. Segunda Edición ed. México: Manual Moderno; 2002.

23. Gasque Gomez R. Enciclopedia Bovina. México D.F: Universidad Nacional Autónoma de México Facultad de Medicina Veterinaria y Zootecnia Ciudad Universitaria México; 2008.

24. Whitlock RH, Buergelt C. Preclinical and clinical manifestations of paratuberculosis (including pathology). Vet Clin North Am Food Anim Pract 1996; 12(2):345-356.

25. Tiwari A, Vanleeuwen JA, Dohoo IR, Keefe GP, Haddad JP, Tremblay R et al. Production effects of pathogens causing bovine leukosis, bovine viral diarrhea, paratuberculosis, and neosporosis. J Dairy Sci 2007; 90(2):659-669.

26. Chiodini RJ, Van Kruiningen $H J$, Merkal RS. Ruminant paratuberculosis (Johne's disease): the current status and future prospects. Cornell Vet $1984 ; 74(3): 218-262$.
27. McKenna SL, Keefe GP, Barkema HW, McClure J, Vanleeuwen JA, Hanna $P$ et al. Cow-level prevalence of paratuberculosis in culled dairy cows in Atlantic Canada and Maine. J Dairy Sci 2004; 87(11):3770-3777.

28. Karcher EL, Beitz DC, Stabel JR. Modulation of cytokine gene expression and secretion during the periparturient period in dairy cows naturally infected with Mycobacterium avium subsp. paratuberculosis. Vet Immunol Immunopathol 2008; 123(34):277-288.

29. Nielsen SS, Enevoldsen C, Grohn YT. The Mycobacterium avium subsp. paratuberculosis ELISA response by parity and stage of lactation. Prev Vet Med 2002; 54(1):1-10.

30. Balseiro A, Prieto JM, Espi A, Perez V, Garcia Marin JF. Presence of focal and multifocal paratuberculosis lesions in mesenteric lymph nodes and the ileocaecal valve of cattle positive to the tuberculin skin test. Vet J 2003; 166(2):210-212.

31. Tiwari A, Vanleeuwen JA, Dohoo IR, Keefe GP, Haddad JP, Scott HM et al. Risk factors associated with Mycobacterium avium subspecies paratuberculosis seropositivity in Canadian dairy cows and herds. Prev Vet Med 2009; 88(1):32-41.

32. Dennis MM, Antognoli MC, Garry FB, Hirst HL, Lombard JE, Gould DH et al. Association of severity of enteric granulomatous inflammation with disseminated Mycobacterium avium subspecies paratuberculosis infection and antemortem test results for paratuberculosis in dairy cows. Vet Microbiol 2008; 131(1-2):154-163. 